Eddy Chukwura Agbo • Birgitta Duim •

Phelix A. O. Majiwa · Philippe Büscher •

Eric Claassen - Marinus F. W. te Pas

\title{
Multiplex-endonuclease genotyping approach (MEGA): a tool for the fine-scale detection of unlinked polymorphic DNA markers
}

Published online: 3 February 2004

(C) Springer-Verlag 2004

\section{Chromosoma (2003) 111:518-524}

The authors have noted that Table 2 of the above paper contained two errors:

i. The 5'-3' MunI adapter oligonucleotide sequence and FAM-labelled MunI primer were shuffled in the print version, which seems to be a type-setting error.

ii. There was inadvertent addition of extra nucleotides ( $\mathrm{T}$ and $\mathrm{G}$ ) to the $B g l \mathrm{II}$ and $\mathrm{MunI}$ primer and adapter sequences in the table by us. The sequences should read ${ }^{5^{\prime}}$-GAG TAC ACT GTC GAT C and ${ }^{5^{\prime}}-6$ FAM-
GAG AGC TCT TGG AAT T, respectively (without the $3^{\prime} \mathrm{T}$ and $\left.\mathrm{G}\right)$.

Since our data were generated with these primers, this correction does not alter the results and conclusions of the paper in any way.

The corrected Table 2 is shown below:

Acknowledgement We thank Drs E. Vansnick and Dirk Geysen (Institute of Tropical Medicine, Antwerp, Belgium) for drawing attention to these points.
Table 2 The complementary sets of BgIII and MunI adapters and polymerase chain reaction primers. (6-FAM is 6-carboxyfluorescein)
Endonuclease

Adapter

5'-CGG ACT AGA GTA CAC TGT C 3'-C TGA TCT CAT GTG ACA GCT AG

(A/GATCT)

Munl

(C/AATTG)
5'- AAT TC CAA GAG CTC TCC AGT AC

3'- G GTT CTC GAG AGG TCA TGA T
Primer core sequence

5'-GAG TAC ACT GTC GAT C

5'-6-FAM-GAG AGC TCT TGG AAT T
The online version of the original article can be found at http:// dx.doi.org/10.1007/s00412-002-0228-y

E. C. Agbo ( $)$

Department of Biological Chemistry,

Johns Hopkins School of Medicine,

725 N. Wolfe St., Baltimore, MD 21205, USA

e-mail: eagbo@jhmi.edu

B. Duim

Department of Medical Microbiology,

University of Amsterdam,

Meibergdreef 15, 1105 AZ Amsterdam, The Netherlands

P. A. O. Majiwa

International Livestock Research Institute,

P.O. Box 30709, Nairobi, Kenya
P. Büscher

Department of Parasitology,

Institute of Tropical Medicine,

Nationalestraat 155, 2000 Antwerp, Belgium

E. Claassen

Department of Immunology,

Erasmus University Rotterdam,

Dr Molewaterplein 50, 3015 GE Rotterdam, The Netherlands

M. F. W. te Pas

Division of Animal Sciences,

Institute for Animal Science and Health,

Edelhertweg 15, 8200 AB Lelystad, The Netherlands 